\title{
Repeat two-stage exchange arthroplasty for recurrent periprosthetic hip or knee infection: what are the chances for success?
}

\author{
A. C. Steinicke ${ }^{1} \cdot$ J. Schwarze ${ }^{1} \cdot$ G. Gosheger $^{1} \cdot$ B. Moellenbeck ${ }^{1} \cdot$ T. Ackmann $^{1} \cdot$ C. Theil ${ }^{1}$ (i)
}

Received: 18 June 2021 / Accepted: 18 December 2021 / Published online: 4 January 2022

(c) The Author(s) 2022

\begin{abstract}
Introduction Two-stage revision is a frequently chosen approach to treat chronic periprosthetic joint infection (PJI). However, management of recurrent infection after a two-stage exchange remains debated and the outcome of a repeat two-stage procedure is unclear. This study investigates the success rates of repeat two-stage exchange arthroplasty and analyzes possible risk factors for failure.

Materials and methods We retrospectively identified 55 patients ( 23 hips, 32 knees) who were treated with repeat resection arthroplasty and planned delayed reimplantation for recurrent periprosthetic joint infection between 2010 and 2019 after a prior two-stage revision at the same institution. The minimum follow-up was 12 months with a median follow-up time of 34 months (IQR 22-51). The infection-free survival, associated revision surgeries, and potential risk factors for further revision were analyzed using Kaplan-Meier survival curves and comparative non-parametric testing.

Results 78\% (43/55) underwent reimplantation after a repeat implant removal. Of those who completed the second-stage surgery, $37 \%$ (16/43) underwent additional revision for infection and 14\% (6/55) underwent amputation. The reinfection-free implant survivorship amounted to 77\% (95\% CI 64-89\%) after 1 year and 38\% (95\% CI 18-57\%) after 5 years. Patients with a higher comorbidity score were less likely to undergo second-stage reimplantation (median 5 vs. 3, $p=0.034$ ). Furthermore, obese patients ( $p=0.026$, Fisher's exact test) and diabetics ( $p<0.001$, log-rank test) had a higher risk for further infection. Most commonly cultures yielded polymicrobial growth at the repeat two-stage exchange $(27 \%, 15 / 55)$ and at re-reinfection $(32 \%, 9 / 28)$. Pathogen persistence was observed in $21 \%$ (6/28) of re-reinfected patients.
\end{abstract}

Conclusion The success rates after repeat two-stage exchange arthroplasty are low. Patients must be counseled accordingly and different modes of treatment should be considered.

Keywords Periprosthetic joint infection $\cdot$ Reinfection $\cdot$ Revision arthroplasty $\cdot$ THA $\cdot$ TKA $\cdot$ Two-stage exchange arthroplasty

\section{Introduction}

Periprosthetic joint infection (PJI) is a severe complication of total joint arthroplasty and occurs in around $1-2 \%$ of primary knee or hip arthroplasties [1]. As the demand for total joint arthroplasty is on the rise due to an aging population,

A. C. Steinicke and J. Schwarze are co-first authors.

C. Theil

christoph.theil@ukmuenster.de

A. C. Steinicke

Amelieconstanze.steinicke@ukmuenster.de

J. Schwarze

jan.schwarze@ukmuenster.de

G. Gosheger

georg.gosheger@ukmuenster.de
B. Moellenbeck

burkhard.moellenbeck@ukmuenster.de

T. Ackmann

Thomas.ackmann@ukmuenster.de

1 Department of Orthopedics and Tumor Orthopedics, Muenster University Hospital, Muenster,

Albert-Schweitzer-Campus 1, 48149 Muenster, Germany 
the revision burden due to PJI is expected to increase as well [1-3].

Two-stage exchange usually using an antibiotic-loaded spacer is considered the gold standard in treating chronic PJI [1]. A two-stage approach with removal of the implant and all foreign material, debridement, and irrigation during first-stage surgery allows for a thorough debridement of all infected tissue and can be considered appropriate for all chronic infection regardless of culture results prior to surgery, soft tissue conditions, and timing of the infection. However, despite these general advantages, two-stage revision surgery is associated with a great deal of morbidity due to the two surgeries required and the period in between stages and the rate of reinfection can be as high as 30\% in some cases [4-10]. Recurrence of infection can be associated with further morbidity as well as a high mortality, particularly if further surgeries are needed [11-13]. One option in these cases is to perform a repeat two-stage procedure, i.e., another sequence of explantation, spacer insertion, and delayed reimplantation of the infected prosthesis [11-13]. Nonetheless, it is debated whether a repeat twostage exchange is adequate, as poor infection-free survival has been reported with failure rates ranging from 22 to $49 \%$ [11-15]. When taken into consideration that many patients do not undergo second-stage reimplantation, failure rates might be even higher [16].

However, despite the high expected rate of reinfection, there is a scarcity of studies on the outcome of a repeat twostage exchange and potential risk factors for failure remain unknown.

This study investigates the success rates of repeat twostage procedures for hip and knee PJI at a single institution, analyzes microbiological findings, and describes possible risk factors for failure.

\section{Material and methods}

The approval of the local ethics committee (2019-042-F-s Ethikkommission der Aerztekammer Westfalen-Lippe und der Westfaelischen-Wilhelms Universitaet Muenster) was obtained before initiation of this retrospective cohort study. Patients were included if they met the following criteria: history of a completed two-stage exchange arthroplasty for chronic hip or knee PJI at our institution, diagnosis of further periprosthetic joint infection of the same joint analog to the criteria published by the Musculoskeletal Infection Society (MSIS) from 2011 [17], treatment with repeat resection arthroplasty and planned delayed reimplantation at our institution between 2010 and 2019, and a minimum followup period of 1 year. However, patients who did undergo revision surgery or died prior to that were included [18]. Patients with prior resection of a bone tumor and subsequent infection were excluded from this study. Using our prospectively maintained institutional joint registry, we identified 305 patients who had undergone two-stage exchange arthroplasty of a hip or knee prosthesis due to chronic PJI at our institution between 2010 and 2017. Of these, 55 were treated with repeat resection arthroplasty and planned delayed reimplantation between 2010 and 2019 due to reinfection. The median follow-up period was 34 months (interquartile range (IQR) 22-51).

Success of the repeated two-stage exchange arthroplasty was defined following the Delphi-based consensus definition that includes healed wounds, no further surgical procedure for infection, and no PJI-related mortality [19].

Data regarding the patients' surgical history, clinical course, medication, and preexisting comorbidities were collected from electronic files. An age-adjusted Charlson Comorbidity Index (CCI) was calculated for each patient [20]. Microbiological findings are presented for the initial two-stage infection, second two-stage, and potential further revisions. Infections were classified as persistent rather than new infections, if at least one pathogen that was cultured at the explantation stage of the preceding two-stage exchange arthroplasty was cultured again at the subsequent two-stage exchange or any further revision for PJI failure [21].

Patient demographics of the study cohort at the time of the repeat two-stage exchange are showcased in Tables 1 and 2. The median age at the time of the repeat two-stage exchange was 73 years (IQR 64-78). An overview of the patients' course and outcomes is provided in Fig. 1.

\section{Surgical approach}

The first stage of a two-stage exchange arthroplasty included the thorough removal of all foreign material and bradytrophic tissue, irrigation, and if needed resection of osteomyelitic bone. Three to seven tissue samples were obtained and sent to our hospital's institute for microbiology, where they were cultured for 7-14 days on Columbia blood agar, Schaedler agar, and Chocolate agar. Concluding the procedure, handmade interim spacers were implanted. Articulating spacers were used for hips, whereas knees underwent implantation of static spacers, both of which were made from polymethylmethacrylate (PMMA) bone cement (Copal $\mathrm{G}+\mathrm{C}$ or Palacos $\mathrm{G}+\mathrm{C}$, Heraeus medical, Wehrheim, Germany) and had $5-10 \%$ of its weight added in antibiotics based on resistance testing (usually $2 \mathrm{~g}$ Vancomycin per $40 \mathrm{~g}$ cement, $4 \mathrm{~g}$ of Meropenem for gram negatives, $600 \mathrm{mg}$ of Voriconazole, or $200 \mathrm{mg}$ of Amphotericin for fungal organisms). In some individual cases, definitive resection arthroplasty of the hip (Girdlestone) was performed, usually if it was likely prior to first-stage surgery that the patient did not wish for any further surgeries due to limited life expectancy, cognitive disorders, or severe comorbidities. 
Table 1 Patient demographics for patients with or without further revision for reinfection after a repeat two-stage exchange
Table 2 Patient demographics for patients with or without further revision for reinfection after a repeat two-stage exchange

\begin{tabular}{lllll}
\hline Variable & Entire study cohort & Reinfection & No reinfection & $\begin{array}{c}p \text { (Fisher's } \\
\text { exact test) }\end{array}$ \\
\hline Female & $49 \%(27 / 55)$ & $48 \%(13 / 27)$ & $52 \%(14 / 27)$ & 0.790 \\
TKA & $58 \%(32 / 55)$ & $53 \%(17 / 32)$ & $47 \%(15 / 32)$ & 0.787 \\
Diabetes mellitus & $40 \%(22 / 55)$ & $68 \%(15 / 22)$ & $32 \%(7 / 22)$ & 0.054 \\
Obesity & $64 \%(35 / 55)$ & $63 \%(22 / 35)$ & $37 \%(13 / 35)$ & $\mathbf{0 . 0 2 6}$ \\
Chronic kidney disease & $27 \%(15 / 55)$ & $53 \%(8 / 15)$ & $47 \%(7 / 15)$ & 1.000 \\
Hypertension & $85 \%(47 / 55)$ & $51 \%(24 / 47)$ & $49 \%(23 / 47)$ & 1.000 \\
Heart disease & $51 \%(28 / 55)$ & $57 \%(16 / 28)$ & $43 \%(12 / 28)$ & 0.423 \\
Depression & $25 \%(14 / 55)$ & $50 \%(7 / 14)$ & $50 \%(7 / 14)$ & 1.000 \\
COPD & $18 \%(10 / 55)$ & $50 \%(5 / 10)$ & $50 \%(5 / 10)$ & 1.000 \\
Anticoagulation & $29 \%(16 / 55)$ & $38 \%(6 / 16)$ & $63 \%(10 / 16)$ & 0.245 \\
Tobacco use & $20 \%(11 / 55)$ & $27 \%(3 / 11)$ & $73 \%(8 / 11)$ & 0.101 \\
Additional DAIR before & $25 \%(14 / 55)$ & $43 \%(6 / 14)$ & $57 \%(8 / 14)$ & 0.547 \\
repeat two-stage revision & & & & \\
\hline
\end{tabular}

${ }^{p}$ values for Fisher's exact test

Significant differences are marked in bold

\begin{tabular}{|c|c|c|c|c|}
\hline Variable & Entire study cohort & Patients reinfected & Patients not reinfected & $\begin{array}{l}p \text { (Mann- } \\
\text { Whitney } U \\
\text { test) }\end{array}$ \\
\hline Age at second PJI & 73 (IQR 64-78) & 72 (IQR 64-78) & 73 (IQR 64-79) & 0.655 \\
\hline Body mass index & 31.4 (IQR 27.7-35.4) & 32 (IQR 30-38) & 29 (IQR 26-33) & 0.040 \\
\hline Age-adjusted CCI & 4 (IQR 3-5) & 4 (IQR 3-5) & 4 (IQR 3-5) & 0.314 \\
\hline $\begin{array}{l}\text { Months between } \\
\text { first and second } \\
\text { PJI }\end{array}$ & 13 (IQR 4-32) & 16 (IQR 5-35) & 10 (IQR 2-26) & 0.162 \\
\hline $\begin{array}{l}\text { Number of previ- } \\
\text { ous surgeries } \\
\text { before second PJI }\end{array}$ & 5 (IQR 4-7) & 5 (IQR 4-7) & 5 (IQR 4-7) & 0.939 \\
\hline
\end{tabular}

${ }^{p}$ values for Mann-Whitney $U$ test

Significant differences are marked in bold
Joint aspiration was not routinely performed before reimplantation given the expected poor sensitivity and specificity [22]. Systemic antibiotic therapy based on the infecting organism was administered for at least two weeks intravenously, followed by four weeks of oral treatment. If inflammatory serum markers (C-reactive protein and interleukin 6) had declined and soft tissues were healed, reimplantation was planned [23]. Eradication of infection was defined based on the criteria defined by Diaz-Ledezma et al. [19] citing healed wounds, absence of PJI-related mortality, and no further revision surgery for infection. For early (within 4 weeks after the last surgery) or acute infections, a DAIR procedure was performed, which includes irrigation, debridement, antibiotic therapy, and the retention of the implant with component exchange, while for later, chronic infections or after a failed debridement procedure, removal of the prosthesis and a repeat staged revision was recommended.
The implants for reimplantation were chosen in consideration of individual factors, such as age, defect size, and bone quality. In three cases, an arthrodesis implant was selected for the second-stage reimplantation. During the reimplantation surgery, deep tissue samples were obtained and sent for microbiological analysis. After reimplantation was completed, antibiotics were administered for 2 weeks in case of negative intraoperative culture results, and for six weeks if positive cultures at the time of reimplantation were obtained.

\section{Statistical analysis}

We pseudonymized all patient data before conducting any statistical analysis.

Descriptive statistics were investigated for data distribution and categorical variables. Means and ranges are used to report parametric data, whereas non-parametric data are 


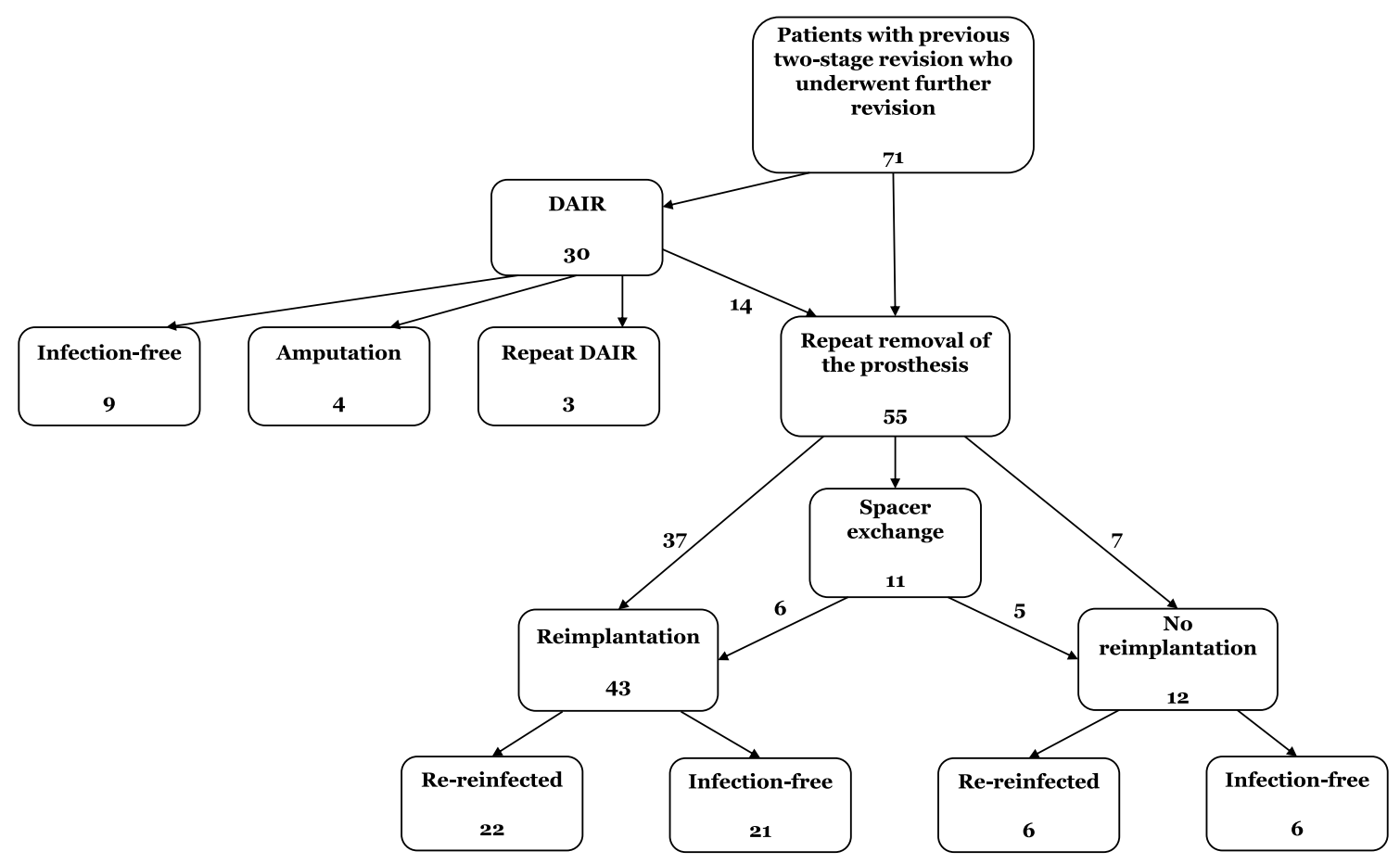

Fig. 1 Flowchart of the treatment course and final outcome

displayed using medians and interquartile ranges (IQR). Differences in groups of binary variables were compared using Fisher's exact test, whereas metric variables were compared using the Mann-Whitney $U$ test for non-parametric distributions or student's $t$ test for parametric distributions of data. Re-reinfection, i.e., failure of the repeat two-stage exchange arthroplasty defined by the Delphi-based criteria [19], was set as the primary outcome measure. Secondary outcome measures were as follows: completion of the repeat two-stage exchange arthroplasty with reimplantation, and amputation.

Implant survival was assessed using the Kaplan-Meier survival analysis with $95 \%$ confidence intervals (CI) presented [24]. Differences in survival were compared using the log-rank test [25]. Again, primary endpoint was the diagnosis of re-reinfection in line with the Delphi consensus criteria [19].

All tests were two tailed with an alpha level of 5\% considered significant.

\section{Results}

\section{Reimplantation rates}

Among the 55 patients who underwent the first stage of a planned repeat two-stage exchange, five knee patients $(16 \%, 5 / 32)$ and seven hip patients $(30 \%, 7 / 23)$ did not complete the two-stage procedure with reimplantation of the prosthesis, making a total of twelve patients $(22 \%$, 12/55) who did not undergo reimplantation. Among these, six are considered reinfected or persistently infected, while in the other six patients the infection is considered eradicated.

Of the six patients who remained infection-free after the explantation stage, two knee patients have died of unrelated cause before the planned reimplantation surgery and four hip patients were successfully treated with a Girdlestone resection arthroplasty due to low functional demand and high surgical risk and remained infection-free until the last follow-up.

Among the patients with persisting infection or reinfection, three patients (two knees, one hip) underwent amputation, one hip patient died from sepsis that was most likely PJI related, one knee patient who was treated with Girdlestone resection arthroplasty underwent a subsequent debridement procedure due to deep tissue reinfection, and one patient with a retained hip spacer had recurrent wound infection.

Patients who did not undergo second-stage reimplantation had a higher median age-adjusted CCI (5 vs. $3(p=0.034)$ ). Furthermore, patients with chronic kidney disease (CKD) $(p=0.068)$ and patients with a shorter time between the first two-stage and reinfection $(p=0.061)$ appeared to be at increased risk to not undergo reimplantation, although no statistical significance could be ascertained with the numbers available. 


\section{Additional surgical performances}

$25 \%(14 / 55)$ of patients ( 8 knees, 6 hips) underwent debridement, irrigation and exchange of the mobile and modular implant components prior to an eventual repeat implant removal. These patients, however, did not have a different outcome than the patients who had not undergone an additional DAIR procedure regarding the rate of re-reinfection after repeat two-stage exchange $(p=0.547)$ or the length of infection-free implant survival ( $p=0.750$ ).

During the prosthesis-free interval of the repeat twostage procedure, ten patients (4 knees, 6 hips) underwent a singular spacer exchange procedure and one hip patient underwent three consecutive spacer exchange procedures, resulting in a total of $20 \%$ of patients $(11 / 55)$ having undergone at least one spacer exchange. The reason for a spacer exchange was spacer dislocation in four cases and clinical signs of persisting infection in nine cases. In our hospital, a spacer exchange due to mechanical reasons, like dislocation, was indicated if the spacer caused pain or disability, threatened neurovascular structures, or could potentially cause skin necrosis, analog to the criteria postulated at the International Consensus Meeting in 2018 [26, 27]. Spacer exchange due to persisting infection was indicated following the Delphi-based consensus criteria [19]. While the cultures taken during spacer exchange were culture negative in $45 \%$ $(5 / 11)$ of spacer exchanges, a different, new organism was cultured during 55\% (6/11) procedures compared to the firststage surgery.

\section{Re-reinfection rates and infection-free implant survival after reimplantation}

Ultimately, 78\% (43/55) of patients underwent reimplantation after a median time of 89 days (IQR 71-143). However, further revision for re-reinfection was performed in $51 \%$ (22/43) of cases (14 knees, 8 hips) after reimplantation. To treat re-reinfection, a total of 15 DAIR procedures, twelve repeat two-stage exchange procedures, and nine amputation surgeries were performed in this subgroup. One patient refused further surgical treatment of his reinfected prosthesis and thus remained with a fistula until last follow-up.

For the 43 cases in which the repeat two-stage procedure was completed, a Kaplan-Meier analysis of the infectionfree implant survival was conducted (Fig. 2). After 1 year, the cumulative infection-free survival probability was $77 \%$ (95\% CI 64-89\%), 65\% after 2 years (95\% CI 50-79\%), and $38 \%$ after 5 years (95\% CI $18-57 \%$ ).

Patients suffering from diabetes mellitus had a significantly lower infection-free survival rate than patients without diabetes mellitus (33\% (95\% CI 9-57\%) vs $82 \%$ (95\% CI $67-96 \%)$ after 2 years, $p<0.001)$.

The infection-free survival was not different between TKA and THA (70\% (95\% CI 53-88\%) vs $56 \%$ (95\% CI $31-80 \%$ ) after 2 years, $p=0.483$ ).

\section{Overall re-reinfection rates, amputation rates, and mortality}

Among all 55 patients who underwent first-stage resection arthroplasty, 51\% (28/55) ultimately suffered from re-reinfection. This includes seven amputation surgeries in the subgroup of knee PJI $(22 \%, 7 / 32)$ and two amputations that were performed on hip patients $(9 \%, 2 / 23)$, resulting in a total amputation rate of $16 \%(9 / 55)$ in our cohort. The overall rate of re-reinfection was not different between hip $(48 \%, 11 / 23)$ and knee $(53 \%, 17 / 32)$ arthroplasties $(p=0.787)$. However, the rate of re-reinfection was significantly higher in obese patients (body mass index $\left.\geq 30 \mathrm{~kg} / \mathrm{m}^{2}\right)(p=0.026)$.
Fig. 2 Kaplan-Meier survival curve for reinfection-free survivorship after completed repeat two-stage revision

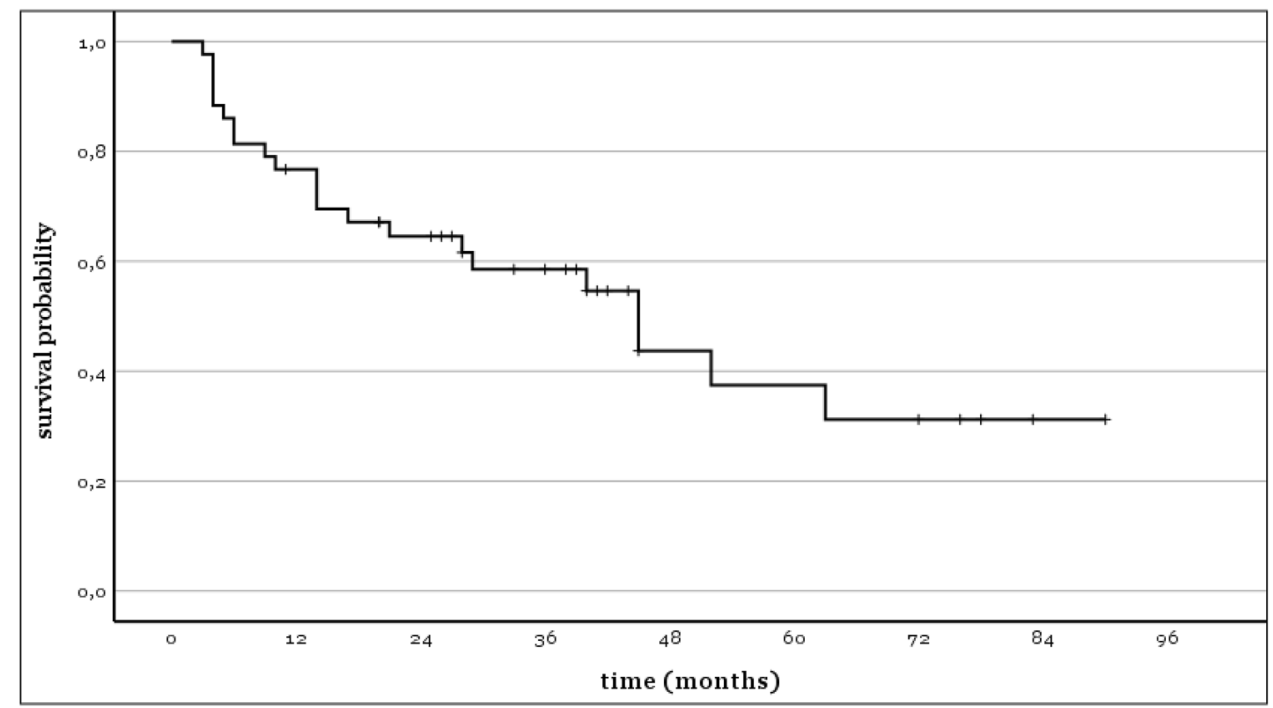


Overall, 29\% (16/55) of patients have died during the follow-up period after a mean time of 32 months (range 1-92). One-year mortality was $11 \%(6 / 55)$ and 30-day mortality was $4 \%(2 / 55)$.

\section{Aseptic complications}

Aseptic complications after the repeat two-stage exchange arthroplasty occurred in seven cases: two patients underwent revision for aseptic TKA loosening, one femoral and tibial each ( 8 and 10 months postoperatively), and five patients suffered hip dislocation and underwent closed reduction in all cases at a median follow-up of 3 months.

\section{Microbiological findings}

The microbiological findings at the explantation stage of the first and the repeat two-stage exchange surgeries and at re-reinfection are presented in Table 3.

While coagulase-negative staphylococci, mostly Staphylococcus epidermidis, were the most common finding during surgery for the first episode of PJI $(31 \%, 17 / 55)$, polymicrobial findings increased with the second and third PJI, being the most reported microbiological finding at reinfection $(27 \%, 15 / 55)$ and re-reinfection $(32 \%, 9 / 28)$. However, with the numbers available, there was no difference in polymicrobial infections between the first, second, and third PJI $(p=0.320)$.

In 9\% (5/55) of all cases (two knees, three hips), the microorganism isolated at the explantation stage of the first two-stage exchange was persistent at the explantation stage of the second two-stage exchange. 67\% (37/55) of reinfections were caused by a new pathogen that had not been isolated at the explantation stage of the first twostage exchange. 24\% (13/55) of the patients in this study cohort were culture negative at either one or both two-stage exchanges or, in two cases, had missing information about the microbiological findings at the first two-stage exchange. The persistence rates among knee patients $(6 \%, 2 / 32)$ and hip patients $(13 \%, 3 / 23)$ do not statistically differ $(p=0.639)$.

Of all 28 patients who are considered re-reinfected after the repeat two-stage exchange arthroplasty, 68\% (19/28) of re-reinfections are considered new infections. A persistent pathogen was observed in $21 \%(6 / 28)$ of re-reinfected patients. Two patients $(7 \%, 2 / 28)$ were culture negative at the second two-stage exchange and one patient's microbiological findings at re-reinfection are missing, as the diagnosis has been made at an outside hospital. Knee patients had a higher persistence rate of $24 \%$ (4/17) than hip patients with a rate of $18 \%(2 / 11)$, however, without a significant statistical difference between the two groups $(p=1.000)$.

$28 \%(13 / 55)$ of patients had positive cultures during reimplantation surgery at the first episode of PJI. During the second two-stage procedure, 37\% (16/43) of patients had positive cultures during reimplantation. However, this difference was not significant between the two episodes $(p=0.182)$.

With the numbers available, no microbiological factors (culture-negative infection, positive culture at reimplantation, or polymicrobial infection) were associated with the risk of re-reinfection or reduced implant survivorship.

\section{Discussion}

Two-stage exchange arthroplasty remains the gold standard for the treatment of chronic periprosthetic joint infection. Still, infection recurrence is observed in $6 \%$ to $33 \%$ of the cases [4-10] and patients suffering from reinfection often face a long course of multiple additional surgeries with a potentially poor outcome [28]. However, given the relatively low number of repeat two-stage exchange arthroplasty procedures reported, data regarding the outcome of patients undergoing this procedure are scarce. This study provides additional data about the success rate of repeat two-stage
Table 3 Causative organisms at each infection

\begin{tabular}{llll}
\hline Organism & $\begin{array}{l}\text { First two-stage } \\
\text { exchange }\end{array}$ & $\begin{array}{l}\text { Second two-stage } \\
\text { exchange }\end{array}$ & Re-reinfection \\
\hline Coagulase-negative staphylococci & $17(31 \%)$ & $14(25 \%)$ & $5(18 \%)$ \\
Staphylococcus aureus & $10(18 \%)$ & $4(7 \%)$ & $4(14 \%)$ \\
Streptococci & $4(7 \%)$ & $4(7 \%)$ & $1(4 \%)$ \\
Gram-negative bacteria & $3(5 \%)$ & $10(18 \%)$ & $5(18 \%)$ \\
Candida spp. & $0(0 \%)$ & $1(2 \%)$ & $0(0 \%)$ \\
Enterococci & $0(0 \%)$ & $1(2 \%)$ & $1(4 \%)$ \\
Anaerobic & $3(5 \%)$ & $0(0 \%)$ & $1(4 \%)$ \\
Culture-negative & $6(11 \%)$ & $6(11 \%)$ & $1(4 \%)$ \\
Polymicrobial & $10(18 \%)$ & $15(27 \%)$ & $9(32 \%)$ \\
Missing & $2(4 \%)$ & $0(0 \%)$ & $1(4 \%)$ \\
Total & $55(100 \%)$ & $55(100 \%)$ & $28(100 \%)$ \\
\hline
\end{tabular}


exchange procedures in a single tertiary revision arthroplasty center and identifies possible risk factors associated with further revision surgery as well as microbiological insights with a high percentage of polymicrobial infections in repeat two-stage procedures and frequent positive cultures during second-stage reimplantation. In our cohort, the probability to remain infection-free amounted to only around $65 \%$ after 2 years and more than half the patients in this cohort ultimately suffered from re-reinfection following this procedure. We found that obese patients and diabetics were at increased risk for further infection and reduced infection-free implant survival, respectively, and patients with a higher comorbidity score were more likely not to undergo second-stage reimplantation.

Prior studies have reported re-reinfection rates after repeat two-stage exchange arthroplasty ranging from 22.2 to $49 \%$ [11-15]. While the comparability to previous studies is somewhat limited by different demographic and surgical details of the patients included, the failure rate presented in our study is relatively high. It should be noted that surgeons must consider various factors in the success of two-stage procedures that are difficult to account for in the retrospective study designs of our and related studies.

Kheir et al. investigated 60 patients who underwent further surgical intervention after a failed two-stage exchange of infected THA or TKA, including 26 patients, with a repeat two-stage procedure that was successful in $62 \%$ of patients [11]. The authors note a higher risk for reinfection after a debridement procedure with retention of the prosthesis compared to a repeat two-stage procedure and propose a more aggressive approach with repeat staged revision. While we also noted that $25 \%$ of patients who ultimately underwent a repeat two-stage procedure had a failed attempt to retain the implant, they did not have a worse outcome after the twostage procedure. Nonetheless, considering that a DAIR procedure is far less invasive than a complete implant removal, we suggest that if early (within 4 weeks) or acute reinfection occurs, patients should be counseled regarding the high risk of failure after a DAIR procedure, but if soft tissue conditions and the patients general state allows for it, it can be an option to avoid further staged revision.

Khan et al. investigated repeat two-stage procedures of infected THA and reported a success rate of $57 \%$ at twoyear follow-up in 42 patients, which is comparable with our study results [12]. With $33 \%$ of patients deceased before the two-year follow-up, the mortality presented in their cohort also corresponds with our results. Khan et al. concluded that an underlying host problem might be the cause for poor outcome regardless of treatment modality and recommended further research on host optimization and other treatment methods for repeat PJI. Considering the equally poor results of repeat two-stage exchanges in our cohort, we agree that patients should be informed regarding this dismal perspective and a mutual decision should be made if further staged revision is planned.

Considering the poor outcome and high risk for re-revision in repeat two-stage procedures in our and the aforementioned studies, there is an urgent need for further investigation on alternative treatment options for repeat PJI.

The retention of a spacer can be a good option in selected patients [29, 30]. Nevertheless, a risk for recurrent infection should be taken into account, particularly if one considers that in the present study, even if no reimplantation is performed, there is a high risk of further revision surgeries for infection.

Amputation is considered a last resort treatment that is usually chosen if the infection cannot be controlled with any other surgical or medical treatment option. The possibility of curing the infection comes at the cost of low functional status and high mortality [31-33]. Nevertheless, amputation can still yield good patient satisfaction [33].

With Girdlestone resection arthroplasty, partial limb function can be preserved, while the infected prosthesis or spacer is removed. However, the rates of complication, mortality, and reoperation are reported to be high [34]. On the other hand, a conversion back to a THA can achieve satisfactory results, if the patient is deemed suitable [35]. This also applies to arthrodesis of the knee [35]. Wu et al. recommended performing arthrodesis as the treatment of choice after a failed two-stage exchange of TKA [36]. In our cohort, only three patients were treated with an arthrodesis instead of a second stage reimplantation and all three subsequently suffered from re-reinfection. Because of the low occurrence in our study, however, we cannot make any general statement concerning the outcome after knee arthrodesis.

Chronic antibiotic suppression can be an option for high surgical risk patients or in cases with large bone defects in which reconstruction would be likely to fail [35]. However, surgeons must consider long-term side effects of suppression therapy and the risk of uncontrolled infection. Furthermore, Leitner et al. investigated the mortality rate of patients with these salvage procedures and found a long-term mortality of $44 \%$ at a median follow-up of 8 years. They recommended a chronic fistula instead of further resection arthroplasty [37]. Regarding repeated revisions in TKA, they recommended considering amputation early after multiple failed revision arthroplasties [37]. Surgeons must always consider that these patients can have a very limited life expectancy and high mortality and further surgical intervention must be weighed regarding the high risk of failure. Nevertheless, if a repeat two-stage exchange for PJI is planned, these patients should also be counseled regarding alternative approaches, but further research is much needed to determine the value of different treatment modalities with regard to reinfection rates and quality of life. 
In order to potentially optimize host factors and assess individual risk, patient- and procedure-related predisposing factors are relevant. However, to our knowledge there are no studies that specifically investigate risk factors in the setting of a repeat two-stage exchange. In the present study, diabetics had a lower implant survival probability. Although this factor has not been described for repeat two-stage revisions, diabetes mellitus is a known risk factor for infection of total hip or knee arthroplasty [38]. Additionally, it has been shown that poorly controlled diabetes appears to be linked to even worse outcomes [39] and in many cases diabetes and obesity are connected [40]. Considering that Fehring et al. reported an increase of obese patients from $30.4 \%$ in 1990 to $52.1 \%$ in 2005 [41], not only the risk for infection after primary arthroplasty but also the risk for further infections must be expected to increase if re-revision is performed. It appears warranted to emphasize diabetes control and to address related complications, such as foot ulcers early on as they might pose as a source of PJI [42]. Future studies should investigate the effect of potential host optimization on the outcome of two-stage procedures and repeat revision.

Polymicrobial infections were most common in patients with repeat two-stage revision but were not associated with a worse outcome. However, the high prevalence of polymicrobial infections must be taken into account to plan the systemic treatment. Contrary to this, Tan et al. found that polymicrobial PJI has a worse outcome and higher PJIrelated mortality than PJI caused by one organism or culturenegative PJI, although this was done in patients with first PJI [43]. In our study cohort, most of the patients suffering rereinfection after the repeat two-stage exchange arthroplasty presented with a new pathogen. Zmistowski et al. suggested that especially patients with poor health status and a high number of comorbidities are at risk for reinfection with new organisms because of their higher disposition to infections rather than due to a failed treatment [21]. However, we found that in this study the percentage of persisting infections increased from the second PJI to re-reinfection. Zmistowski et al. found that Staphylococcal organisms, especially methicillin-resistant Staphylococcus aureus, have a high risk to be persistent in recurrent PJI. Further research on pathogen persistence rates, microbiological resistance patterns, and antimicrobial treatment could provide interesting additional insight.

This study's findings must be interpreted considering several limitations: Our study data were collected at a single institution and therefore might include bias that comes from local preferences or routines. There may have also been a selection bias since our study cohort only consists of patients who were deemed fit enough for a repeat two-stage exchange arthroplasty; however, in our practice generally further surgery was recommended if reinfection was present. Nonetheless, it is possible that patients had undergone surgery elsewhere or that suppression treatment has been initiated. Furthermore, for some statistical analysis there might be sparse data bias as the number of risk factors and number of events are limited and a multivariate analysis is not helpful in this situation. Nevertheless, we think that our findings are a valuable addition to the existing research on this rare issue.

\section{Conclusion}

Repeat two-stage revision for recalcitrant infection after a two-stage procedure for PJI leads to further infection in around $50 \%$ of patients at mid-term follow-up. Particularly, obese patients and diabetics are at high risk of reinfection. Surgeons should be aware of the high percentage of polymicrobial infections encountered at a repeat two-stage procedure and plan antibiotic treatment accordingly. Furthermore, particularly in comorbid patients an additional share of patients did not reach second-stage reimplantation; therefore, alternative strategies should be discussed in order to avoid re-revisions in this challenging group of patients.

Author contributions ACS contributed to conception and design, acquisition of data, analysis and interpretation of data, and drafting of the manuscript; JS was involved in review of literature, drafting of the manuscript, and conception and design; GG contributed to conception and design, supervision and expert advice, and critical revision of the manuscript; $\mathrm{BM}$ and TA performed conception and design, analysis and interpretation of data, drafting of the manuscript, and critical revision of the manuscript; ; CT performed review of literature, conception and design, acquisition of data, analysis and interpretation of data, statistical analysis, and drafting of the manuscript. All the authors read and approved the final manuscript.

Funding Open Access funding enabled and organized by Projekt DEAL. No funding was received.

Availability of data and material The datasets used and/or analyzed during the current study are available from the corresponding author upon reasonable request.

\section{Declarations}

Conflict of interest The authors have no relevant financial or non-financial interests to disclose.

Ethical approval The authors had an ethics approval of the local ethics committee, reference number 2019-042-F-s (Ethikkommission der Aerztekammer Westfalen-Lippe und der Westfaelischen-Wilhelms Universitaet Muenster). Consent was not deemed necessary by the ethics committee because this study is a retrospective analysis of routine clinical data.

Consent for publication Consent for publication was not necessary because the manuscript does not contain individual data. 
Open Access This article is licensed under a Creative Commons Attribution 4.0 International License, which permits use, sharing, adaptation, distribution and reproduction in any medium or format, as long as you give appropriate credit to the original author(s) and the source, provide a link to the Creative Commons licence, and indicate if changes were made. The images or other third party material in this article are included in the article's Creative Commons licence, unless indicated otherwise in a credit line to the material. If material is not included in the article's Creative Commons licence and your intended use is not permitted by statutory regulation or exceeds the permitted use, you will need to obtain permission directly from the copyright holder. To view a copy of this licence, visit http://creativecommons.org/licenses/by/4.0/.

\section{References}

1. Kapadia BH, Berg RA, Daley JA et al (2016) Periprosthetic joint infection. Lancet 387:386-394. https://doi.org/10.1016/ S0140-6736(14)61798-0

2. Murphy BPd, Dowsey MM, Spelman T et al (2018) The impact of older age on patient outcomes following primary total knee arthroplasty. Bone Joint J 100-B:1463-1470. https://doi.org/10. 1302/0301-620X.100B11.BJJ-2017-0753.R6

3. Helwig P, Morlock J, Oberst M et al (2014) Periprosthetic joint infection-effect on quality of life. Int Orthop 38:1077-1081. https://doi.org/10.1007/s00264-013-2265-y

4. Berend KR, Lombardi AV Jr, Morris MJ et al (2013) Twostage treatment of hip periprosthetic joint infection is associated with a high rate of infection control but high mortality. Clin Orthop Relat Res 471:510-518. https://doi.org/10.1007/ s11999-012-2595-x

5. Wolf M, Clar H, Friesenbichler J et al (2014) Prosthetic joint infection following total hip replacement: results of one-stage versus two-stage exchange. Int Orthop 38:1363-1368. https:// doi.org/10.1007/s00264-014-2309-y

6. Pangaud C, Ollivier M, Argenson J-N (2019) Outcome of single-stage versus two-stage exchange for revision knee arthroplasty for chronic periprosthetic infection. EFORT Open Rev 4:495-502. https://doi.org/10.1302/2058-5241.4.190003

7. Kunutsor SK, Whitehouse MR, Lenguerrand E et al (2016) ReInfection outcomes following one- and two-stage surgical revision of infected knee prosthesis: a systematic review and metaanalysis. PLoS ONE 11:e0151537. https://doi.org/10.1371/journ al.pone. 0151537

8. Kunutsor SK, Whitehouse MR, Blom AW et al (2015) Re-infection outcomes following one- and two-stage surgical revision of infected hip prosthesis: a systematic review and meta-analysis. PLoS ONE 10:e0139166. https://doi.org/10.1371/journal.pone. 0139166

9. Sabry FY, Buller L, Ahmed S et al (2014) Preoperative prediction of failure following two-stage revision for knee prosthetic joint infections. J Arthroplasty 29:115-121. https://doi.org/10. 1016/j.arth.2013.04.016

10. Barton CB, Wang DL, An Q et al (2020) Two-stage exchange arthroplasty for periprosthetic joint infection following total hip or knee arthroplasty is associated with high attrition rate and mortality. J Arthroplasty 35:1384-1389. https://doi.org/10. 1016/j.arth.2019.12.005

11. Kheir MM, Tan TL, Gomez MM et al (2017) Patients with failed prior two-stage exchange have poor outcomes after further surgical intervention. J Arthroplasty 32:1262-1265. https://doi.org/ 10.1016/j.arth.2016.10.008

12. Khan N, Parmar D, Ibrahim MS et al (2019) Outcomes of repeat two-stage exchange hip arthroplasty for prosthetic joint infection. Bone Joint J 101-B:110-115. https://doi.org/10.1302/ 0301-620X.101B6.BJJ-2018-1556.R1

13. Azzam K, McHale K, Austin M et al (2009) Outcome of a second two-stage reimplantation for periprosthetic knee infection. Clin Orthop Relat Res 467:1706-1714. https://doi.org/10.1007/ s11999-009-0739-4

14. Brown TS, Fehring KA, Ollivier M et al (2018) Repeat twostage exchange arthroplasty for prosthetic hip re-infection. Bone Joint J 100-B:1157-1161. https://doi.org/10.1302/0301-620X. 100B9.BJJ-2018-0470.R1

15. Fehring KA, Abdel MP, Ollivier M et al (2017) Repeat twostage exchange arthroplasty for periprosthetic knee infection is dependent on host grade. J Bone Joint Surg Am 99:19-24. https://doi.org/10.2106/JBJS.16.00075

16. Gomez MM, Tan TL, Manrique J et al (2015) The fate of spacers in the treatment of periprosthetic joint infection. J Bone Joint Surg Am 97:1495-1502. https://doi.org/10.2106/JBJS.N.00958

17. Parvizi J, Zmistowski B, Berbari EF et al (2011) New definition for periprosthetic joint infection: from the Workgroup of the Musculoskeletal Infection Society. Clin Orthop Relat Res 469:29922994. https://doi.org/10.1007/s11999-011-2102-9

18. Xu C, Tan TL, Li WT et al (2020) Reporting outcomes of treatment for periprosthetic joint infection of the knee and hip together with a minimum 1-year follow-up is reliable. J Arthroplasty 35:1906-1911.e5. https://doi.org/10.1016/j.arth.2020.02.017

19. Diaz-Ledezma C, Higuera CA, Parvizi J (2013) Success after treatment of periprosthetic joint infection: a Delphi-based international multidisciplinary consensus. Clin Orthop Relat Res 471:2374-2382. https://doi.org/10.1007/s11999-013-2866-1

20. Yang C-C, Fong Y, Lin L-C et al (2018) The age-adjusted Charlson comorbidity index is a better predictor of survival in operated lung cancer patients than the Charlson and Elixhauser comorbidity indices. Eur J Cardiothorac Surg 53:235-240. https://doi.org/10. 1093/ejcts/ezx215

21. Zmistowski B, Tetreault MW, Alijanipour P et al (2013) Recurrent periprosthetic joint infection: persistent or new infection? J Arthroplasty 28:1486-1489. https://doi.org/10.1016/j.arth.2013. 02.021

22. Hoell S, Moeller A, Gosheger G et al (2016) Two-stage revision arthroplasty for periprosthetic joint infections: what is the value of cultures and white cell count in synovial fluid and CRP in serum before second stage reimplantation? Arch Orthop Trauma Surg 136:447-452. https://doi.org/10.1007/s00402-015-2404-6

23. Hoell S, Borgers L, Gosheger G et al (2015) Interleukin-6 in two-stage revision arthroplasty: what is the threshold value to exclude persistent infection before re-implanatation? Bone Joint J 97-B:71-75. https://doi.org/10.1302/0301-620X.97B1.33802

24. Kaplan EL, Meier P (1958) Nonparametric estimation from incomplete observations. J Am Stat Assoc 53:457-481. https:// doi.org/10.1080/01621459.1958.10501452

25. Mantel N (1966) Evaluation of survival data and two new rank order statistics arising in its consideration. Cancer Chemother Rep 50:163-170

26. Kozaily E, Chisari E, Parvizi J (2020) Is there a role for spacer exchange in two-stage exchange arthroplasty for periprosthetic joint infection? J Clin Med. https://doi.org/10.3390/jcm9092901

27. Abdel MP, Barreira P, Battenberg A et al (2019) Hip and knee section, treatment, two-stage exchange spacer-related: proceedings of international consensus on orthopedic infections. J Arthroplasty 34:427-438

28. Shahi A, Tan TL, Chen AF et al (2017) In-hospital mortality in patients with periprosthetic joint infection. J Arthroplasty 32:948952.e1. https://doi.org/10.1016/j.arth.2016.09.027

29. Choi H-R, Freiberg AA, Malchau H et al (2014) The fate of unplanned retention of prosthetic articulating spacers for infected 
total hip and total knee arthroplasty. J Arthroplasty 29:690-693. https://doi.org/10.1016/j.arth.2013.07.013

30. Lee K-J, Min B-W, Bae K-C et al (2020) Unintended retention of temporary articulating spacers in the treatment of periprosthetic hip joint infection. Orthopedics 43:e251-e257. https://doi.org/10. 3928/01477447-20200404-08

31. Böhle S, Rohe S, Kirschberg J et al (2020) Poor outcome of aboveknee amputation after septic failure of revision total knee arthroplasty. J Knee Surg. https://doi.org/10.1055/s-0040-1716416

32. Fedorka CJ, Chen AF, McGarry WM et al (2011) Functional ability after above-the-knee amputation for infected total knee arthroplasty. Clin Orthop Relat Res 469:1024-1032. https://doi. org/10.1007/s11999-010-1577-0

33. Orfanos AV, Michael RJ, Keeney BJ et al (2020) Patient-reported outcomes after above-knee amputation for prosthetic joint infection. Knee 27:1101-1105. https://doi.org/10.1016/j.knee.2019.10. 007

34. Malcolm TL, Gad BV, Elsharkawy KA et al (2015) Complication, survival, and reoperation rates following girdlestone resection arthroplasty. J Arthroplasty 30:1183-1186. https://doi.org/ 10.1016/j.arth.2015.02.011

35. Fagotti L, Tatka J, Salles MJC et al (2018) Risk factors and treatment options for failure of a two-stage exchange. Curr Rev Musculoskelet Med 11:420-427. https://doi.org/10.1007/ s12178-018-9504-1

36. Wu CH, Gray CF, Lee G-C (2014) Arthrodesis should be strongly considered after failed two-stage reimplantation TKA. Clin Orthop Relat Res 472:3295-3304. https://doi.org/10.1007/ s11999-014-3482-4

37. Leitner L, Posch F, Amerstorfer F et al (2020) The dark side of arthroplasty: competing risk analysis of failed hip and knee arthroplasty with periprosthetic joint infection. J Arthroplasty 35:26012606.e1. https://doi.org/10.1016/j.arth.2020.04.078
38. Maradit Kremers H, Lewallen LW, Mabry TM et al (2015) Diabetes mellitus, hyperglycemia, hemoglobin $\mathrm{A} 1 \mathrm{C}$ and the risk of prosthetic joint infections in total hip and knee arthroplasty. J Arthroplasty 30:439-443. https://doi.org/10.1016/j.arth.2014.10. 009

39. Wang S-H, Xu C, Tan TL et al (2020) Increased postoperative glucose variability is associated with adverse outcome following two-stage exchange arthroplasty for periprosthetic joint infection. J Arthroplasty 35:1368-1373. https://doi.org/10.1016/j.arth.2019. 11.046

40. Dowsey MM, Choong PFM (2009) Obese diabetic patients are at substantial risk for deep infection after primary TKA. Clin Orthop Relat Res 467:1577-1581. https://doi.org/10.1007/ s11999-008-0551-6

41. Fehring TK, Odum SM, Griffin WL et al (2007) The obesity epidemic: its effect on total joint arthroplasty. J Arthroplasty 22:7176. https://doi.org/10.1016/j.arth.2007.04.014

42. Rakow A, Perka C, Trampuz A et al (2019) Origin and characteristics of haematogenous periprosthetic joint infection. Clin Microbiol Infect 25:845-850. https://doi.org/10.1016/j.cmi.2018. 10.010

43. Tan TL, Kheir MM, Tan DD et al (2016) Polymicrobial periprosthetic joint infections: outcome of treatment and identification of risk factors. J Bone Joint Surg Am 98:2082-2088. https://doi.org/ 10.2106/JBJS.15.01450

Publisher's Note Springer Nature remains neutral with regard to jurisdictional claims in published maps and institutional affiliations. 